\title{
Asymmetric Dependence Analysis of International Crude Oil Spot and Futures Based on the Time Varying Copula-GARCH
}

\author{
$\mathrm{He} \mathrm{Xin}^{1, *}$ and Zhang Jun ${ }^{2}$ \\ ${ }^{I}$ Department of Information Management, Beijing Institute of Petrochemical Technology, Beijing, 102617, P.R. China; \\ ${ }^{2}$ Department of Information Management, Tianjin University of Science and Technology, Tianjin, 300457, P.R. China
}

\begin{abstract}
Taking daily return of international crude oil spot and futures as sample, this paper analyzed the time varying and asymmetric dependence structure of them by time varying Copula-GARCH model based on sliding window and semi parameter estimation. This paper analyzed the regular changing between dependence structure of crude oil spot and futures and the return fluctuation, and confirmed that there is significant time varying asymmetric tail dependence. This paper found that the size of the sliding window had no significant influence on the conclusion, and the data of weekly return is more suitable for analysis of the trend of dependence structure of spot.
\end{abstract}

Keywords: Crude oil market, Dependence structure, Futures price, Sliding window, Time-varying copulas.

\section{INTRODUCTION}

Over the years, there have been many researches on the complicated dependence structure of spot and futures trading of crude oil on the international market. The findings have been used in risk analysis and hedge operations. From the perspective of existing research results, most research used the same analysis methods, in spite of the different focuses of researches around the world.

Arouri et al., [1] structured a variety of GARCH models to analyze conditional volatilities of spot and futures prices of crude oil with structural break and long memory, concluding that the approach has better forecast ability than existing ones. Using a mixed model of Copula and ARJI-GARCH, Chang [2] studied the asymmetric, time-varying dependence of oil spot and futures prices, and concluded that the ARJIGARCH is a better choice for the analysis of non-continuous data than the traditional AR-GARCH model. In their study about the volatility structure of international oil futures prices, Chiarella et al., [3] discovered a Bell Curve in the price changes. In a study about the structural break between the oil spot and futures markets, Chen et al., [4] performed cointegration and Granger Causality tests, finding that structural break has a significant impact on long-term co-integration and causality.

In China, most research focuses on the spillover effect of international oil price shocks on the domestic stock market, while some are also concerned with analysis on the interdependence of spot and futures prices. Basically, the same approaches of foreign peers are used in these studies. G. Guofeng and Z. Zhaofeng [5] used the GARCH(1,1)-M model in a study on the impact of international energy

*Address correspondence to this author at the Department of information management, Beijing Institute of Petrochemical Technology, Beijing, 102617, P.R. China; Tel: +86 10 52489515; Fax: +86 10 58850501-1987; E-mail: hexin@bipt.edu.cn price shock on China's stock market, concluding price shock on China's stock market, concluding that oil price shocks have significant spillover effect on the Shanghai/Shenzhen Stock Markets. Xiaohua et al., [6] used an EGARCH-EVT-t Copula-based measurement model to study oil price risks in the world market, and discovered that the absolute risk in the oil market increases along with the confidence level and contract duration. In his study about the optimal hedge ratio in the gold market, X. Chi [7] used the M-Copula-GJR-VaR model, which turned out to be one of the most frequently used models in oil spot and futures studies.

This paper focuses on the asymmetric, time varying dependence between spot and futures prices of crude oil in the international market. Compared with existing studies, it has two distinct features. First, unlike most existing studies, which use the DCC-Copula model for time varying dependence analysis. This paper uses the sliding window approach, along with time varying Copula to estimate the time varying dependence coefficients and tail dependence coefficients of oil spot and futures prices, while analyzing the impact of different time windows on these results. Second, while weekly or monthly return is adopted in most existing studies, this paper uses daily return in its modeling and analysis, while evaluating the impact of daily return on the result of analysis, as compared with that of weekly return.

\section{EMPIRICAL RESEARCH MODEL}

As the first step of the study, we developed GARCH models for daily returns of oil spot and futures markets using the marginal distribution modeling approach. Then through combined distribution modeling, we estimated the time varying Copula parameters of the standard residuals. Finally, we calculated the time varying dependence coefficients and tail dependence coefficients. 


\subsection{Marginal Distribution Estimation Model}

Bollerslev's [8] standard GARCH $(1,1)$ model is the most frequently used model for the analysis of financial time series volatilities. In this study, we discovered that it would be more suitable to develop a ARMA $(m, n)-G A R C H ~(1,1)$ model for the oil spot and futures return series. With given time series, the ARMA $(\mathrm{m}, \mathrm{n})-\operatorname{GARCH}(1,1)$ model is described as:

$y_{t}=\mu+\sum_{i=1}^{m} a_{i} y_{t-i}+\sum_{j=1}^{n} b_{j} \varepsilon_{t-j}+\varepsilon_{t}$

$\varepsilon_{t}=\sigma_{t} z_{t}$

$\sigma_{t}^{2}=\omega_{0}+\alpha \varepsilon_{t-1}^{2}+\beta \sigma_{t-1}^{2}$

where is an independent and identically distributed series with 0 mean and 1 variance, while is the conditional variance of time $t$ in the time series. The conditional variance process requires ,, and .

In this study, the AIC and BIC criteria are used to determine the optimal lag orders $m$ and $n$.

\subsection{The Copula Function}

The Copula function is the most extensively used tool in financial time series dependence modeling. Its biggest benefit lies in the fact that it separates marginal distribution modeling from combined modeling, and therefore provides the flexibility to consider the clustering effect of return series and the complexity of the dependence structure at the same time.

An n-dimensional Copula is a multi-dimensional distribution function $\mathrm{C}$ with standard uniform marginal distribution. The Sklar Theorem ensures the existence of the Copula function.

\section{The Sklar Theorem}

Let be random variants with marginal distributions, and their combined distribution be $\mathrm{H}$. Then there exists a Copula function that allows:

$$
H\left(x_{1}, \cdots, x_{d}\right)=C\left(F_{1}\left(x_{1}\right), \cdots, F_{d}\left(x_{d}\right)\right)
$$

Reversely, if $C$ is a Copula function and $F_{1}, \cdots, F_{d}$ distribution functions, then the above function $H$ is a combined distribution with marginal distributions $F_{1}, \cdots, F_{d}$.

In empirical financial studies, the most frequently used Copula functions include normal Copula, t Copula, Clayton Copula, Gumbel Copula, FGM Copula and extreme value Copula.

\subsection{Time Varying Copula Functions}

Patton [9] was the first one to study time varying Copula functions. Patton proposed to use a process similar to AR$\mathrm{MA}(1,10)$ to describe parameter of 2-dimensional normal Copula. Since then, it has been modified by many researchers. Today, the most frequently used approach in empirical studies is to set 2-dimensional normal Copula as DCC.
As 2-dimensional normal Copula cannot effectively describe the complicated dependence structure of financial time series, the sliding window approach is used in this study, along with the Canonical Maximum Likelihood approach to estimate the normal Copula, Clayton Copula and Gumbel Copula parameters

\subsection{Dependence Measurement}

In view of the vulnerabilities of dependence coefficient, rank correlation coefficient Kendall is used in this study to measure the dependence of oil spot and futures markets, along with the tail dependence coefficient for the measurement in extreme scenarios.

The rank correlation coefficient of time series $\mathrm{X}$ and $\mathrm{Y}$ is defined as:

$$
\begin{aligned}
& \tau(X, Y)=\operatorname{Pr}\left(\left(X_{1}-X_{2}\right)\left(Y_{1}-Y_{2}\right)>0\right) \\
& -\operatorname{Pr}\left(\left(X_{1}-X_{2}\right)\left(Y_{1}-Y_{2}\right)<0\right)
\end{aligned}
$$

Upper/lower tail dependence coefficients are defined as:

$$
\begin{aligned}
& \lambda_{U}=\lim _{u \rightarrow 1^{-}} \operatorname{Pr}\left(F_{1}(X)>u \mid F_{2}(Y)>u\right) \\
& \lambda_{L}=\lim _{u \rightarrow 0^{+}} \operatorname{Pr}\left(F_{1}(X)<u \mid F_{2}(Y)<u\right)
\end{aligned}
$$

Common Copula functions have one-for-one correlations with Kendall and tail dependence coefficients, but that is not in the scope of this study.

\section{RESULTS OF EMPIRICAL RESEARCH}

\subsection{Data and Statistical Identities}

Daily spot and futures prices of WTI in the time window of January 2005 to July 2014 were collected from the official website of US Energy Information Administration (EIA). Then these were converted into daily return data. Fig. (1) shows the return series and the return mean square series, with obvious volatility clustering and series dependence identities. Table 1 provides descriptive statistics of the return series, indicating that neither is obviously normal. The linear dependence coefficient of oil spot and futures return series is 0.9313 .

\subsection{Marginal Distribution Estimation}

Through LB test, we found significant dependence in both return series. During the ARMA modeling process, we discovered in the test that the parameters have significant ARCH. Finally, based on the result of goodness-of-fit test, we estimated both return series to be ARMA $(2,2)$ $\operatorname{GARCH}(1,1)$ models.

\subsection{Time Varying Copula Model Estimation}

Having extracted standard residuals from the marginal distributions, we estimated the Copula functions with the ECDF approach, which was originally proposed by Chen et al., $[10,11]$ for the calculation of semi-parameters of densities. We estimated the time varying parameters of the Gumbel Copula, Clayton Copula and normal Copula. In order to 
compare the results of estimation, we converted the parameters into Kendall'. Fig. (2) shows Kendall Tau with a 120day gliding window.
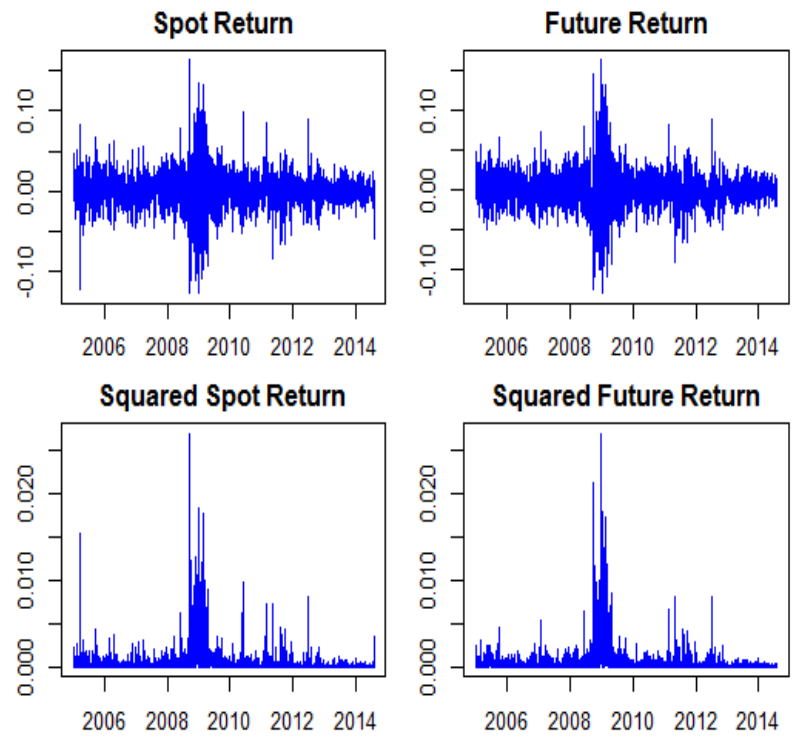

Fig. (1). Daily returns of oil spot and futures markets.

Table 1. Descriptive statistics.

\begin{tabular}{|c|c|c|c|c|c|c|}
\hline & High & Low & Mean & SD & Deviation & Kurtosis \\
\hline \hline Spot & -0.1283 & 0.1641 & 0.00035 & 0.02321 & 0.0418 & 5.8091 \\
\hline Futures & -0.1307 & 0.1641 & 0.00035 & 0.022943 & 0.1321 & 5.7488 \\
\hline
\end{tabular}

From Fig. (2) we can see a complicated time varying dependence structure between oil spot and futures markets. In general, the three Copulas have the same change pattern. For example, during the 2006-2009 time frame, when oil spot and futures returns were highly volatile, their rank correlation coefficients increased. In the 2009-2010 time frame, when oil spot and futures returns were less volatile, the rank correlation coefficients dropped significantly. Since 2010, as the returns stabilized, there has been a close correlation between the two.

Rank correlation coefficients obtained through a normal Copula are always lower than those obtained through the Gumbel Copula and Clayton Copula, especially in 20082009 , when the returns were most volatile, as a normal Copula significantly underestimates their correlation. Therefore, a normal Copula is not suitable for the analysis of the time varying dependence of oil spot and futures markets, as the results would underestimate dependence risks.

\subsection{Estimation of Time Varying Tail Dependence Coeffi- cients}

Next, we estimated upper tail dependence coefficient with a Gumbel Copula and lower tail dependence coefficient with a Clayton Copula. Fig. (3) shows tail dependence coefficients with a 120-day sliding window.
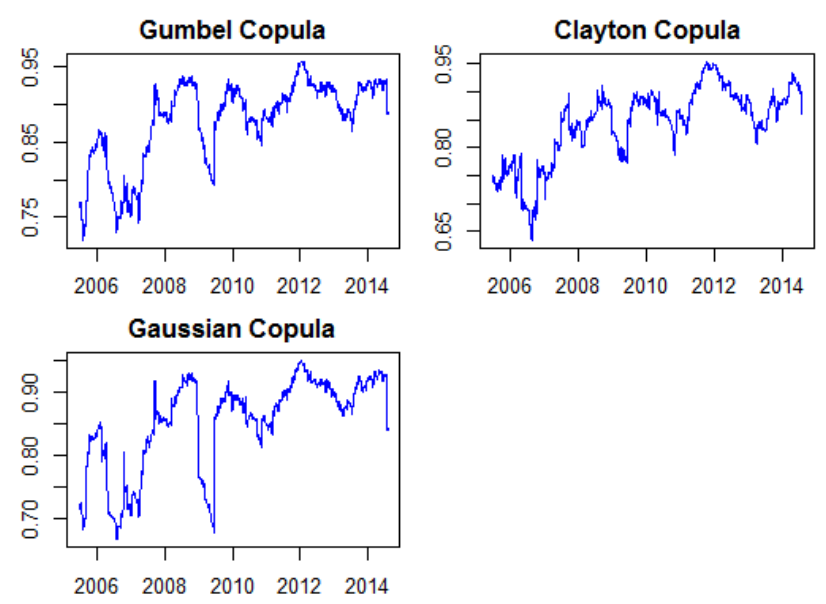

Fig. (2). Kendall Tau with a 120-day gliding window.


Fig. (3). Tail dependence coefficients with a 120-day sliding window.

From Fig. (3) we can see that there is obvious asymmetric tail dependence between oil spot and futures markets, and that lower tails are higher than upper tails. It means that the dependence is stronger in times of significant decline of returns. In other words, the dependence risk is higher in times of a significant price drop.

\section{ROBUSTNESS ANALYSIS FOR THE APPROACH}

Next, we verified the sensitivity of the time varying Copulas approach to the size of the sliding window and data collection frequency.

\subsection{The Impact of Sliding Window Size}

We re-estimated the parameters of the three Copulas function with a 250-day window (annual), and converted them into Kendall (see Fig. 4). The rank correlation coefficients obtained are basically consistent with those shown in Fig. (2), only with slightly smoother curves. Obviously, the size of the sliding window does not have a significant impact on the time varying dependence structure.

Fig. (5) provides tail dependence coefficients with a 250day sliding window. The results are consistent with those in Fig. (4), only with higher estimated values. Obviously, larger sliding windows are helpful for the calculation and analysis of tail risks.

\subsection{Impact of Weekly Return Data}

In order to ensure consistency and comparability with similar researches, we used price data of the same time windows, and converted them into weekly return data. Using the 
same approach, we calculated time varying dependence coefficients (see Fig. 6), which tend to be higher than those in Fig. (2), along with more obvious trend curves. Particularly, there is a notable pattern of gradually increasing dependence between oil spot and futures markets in the wake of drastic return volatilities. Obviously, the weekly return-based approach is more adequate to identify the change pattern of the dependence structure.
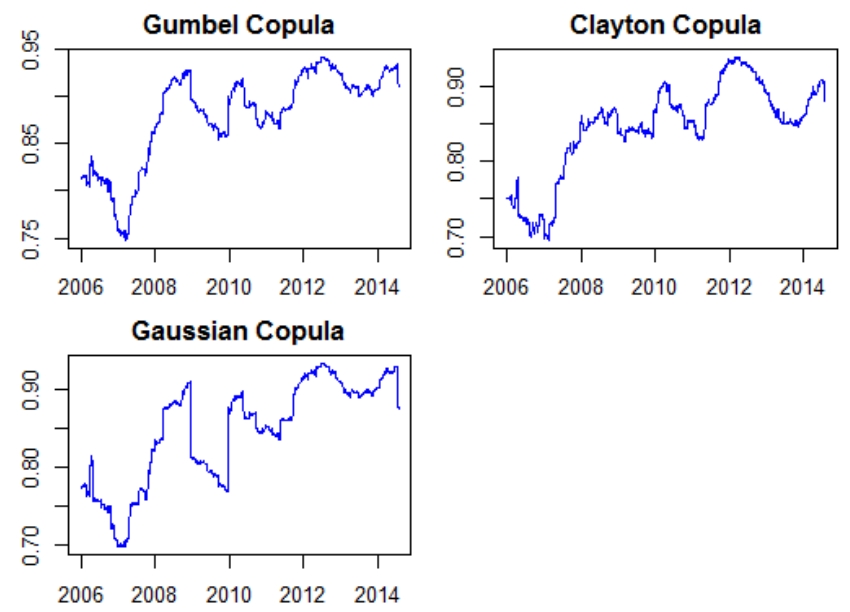

Fig. (4). Kendall Tau with a 250-day gliding window.
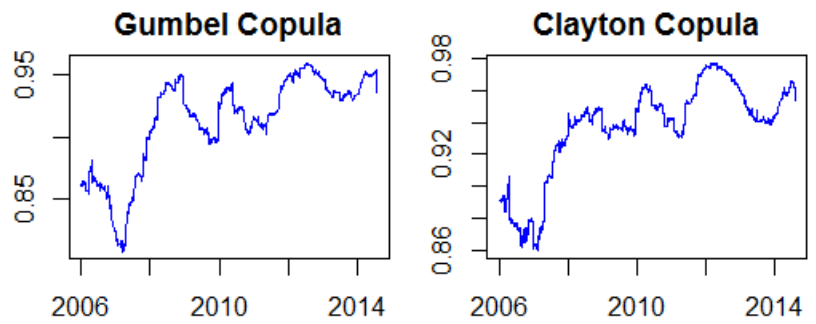

Fig. (5). Tail dependence coefficients with a 250-day sliding window.
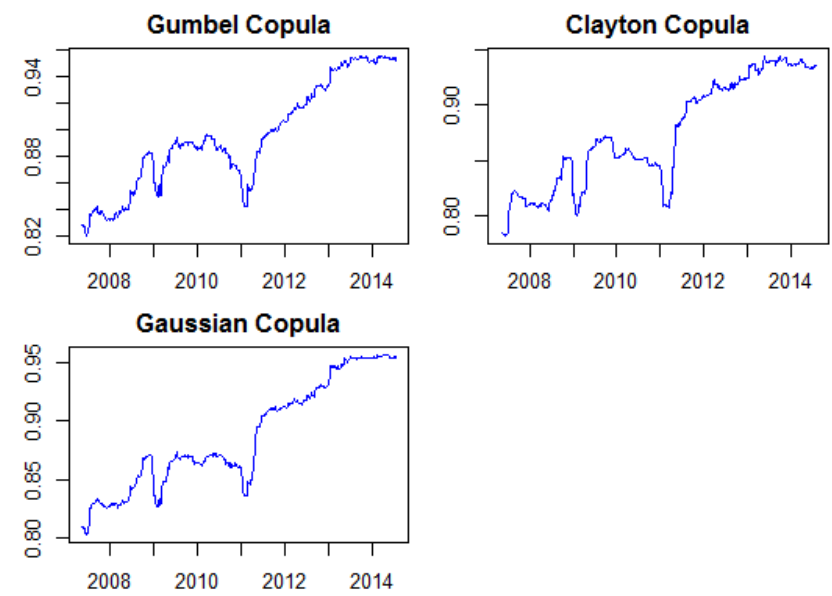

Fig. (6). Kendall Tau of weekly returns with a 120-day gliding window.

All of the tail dependence coefficients in Fig. (7) are higher than those in Fig. (2), along with notable asymmetric and trend identities.
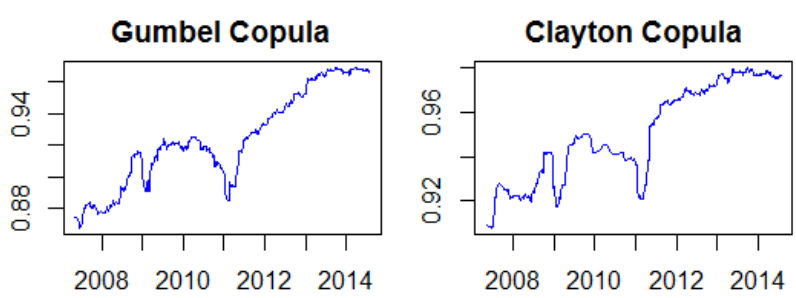

Fig. (7). Tail dependence coefficients of weekly returns with a 120day gliding window.

\section{CONCLUSION}

In the study, we found that:

1) There is a complicated time varying dependence structure between the oil spot and futures markets. Their rank correlation coefficients would rise significantly in times of high volatilities of returns, and decrease as the volatilities decline. In the meantime, there is a asymmetric tail dependence relation between the two, with lower tail coefficients higher the upper tail coefficients. It means that oil spot and futures markets are more sensitive to significant price drops.

2) A normal Copula is not suitable for the analysis of the time varying dependence of oil spot and futures markets as it significantly underestimates their correlation. Particularly, incapable of measuring tail dependence, it would increase risks to hedge operations.

3) Rank correlation coefficients calculated with weekly return data tend to be higher than those with weekly return data, along with smoother changes and more obvious trend curves. Therefore, while daily return-based data are useful for more accurate estimation of dependence risks, weekly return-based data are more suitable for dependence structure and trend analysis.

One weakness of this study is that it uses only a Gumbel Copula for the description of upper tail dependence and a Clayton Copula for lower tail dependence. During the study, we found significant asymmetric tail dependence between oil spot and futures market. Therefore, we need a better Copula function to describe it.

In the follow-on studies, we are going to test the results of multiple time varying Copula functions, while considering the impact of structural break. In the meantime, we are going to apply the findings of this study in the calculation of time varying hedge operation ratios.

\section{CONFLICT OF INTEREST}

The authors confirm that this article content has no conflict of interest.

\section{ACKNOWLEDGEMENTS}

The research work was supported by National Natural Science Foundation of China under Grant No. 71071022 and Tianjin Municipal Philosophy and Social Science Research Plan Project under Grant No. TJGL13-042. 


\section{REFERENCES}

[1] M. Arouri, A. Lahiani, A. Lévy, and D.K. Nguyen, "Forecasting the conditional volatility of oil spot and futures prices with structural breaks and long memory models," Energy Economics, vol. 34, pp. 283-293, 2012 .

[2] K.L. Chang, "The time-varying and asymmetric dependence between crude oil spot and futures markets: Evidence from the mixture copula ARJI-GARCH model," Economic Modeling, vol. 29, pp. 2298-2309, 2012.

[3] C. Chiarella, B. Kang, and C.S. Nikitopoulos, "Thy-Duoong Tô. Humps in the volatility structure of the crude oil futures market: New evidence," Energy Economics, vol. 40, pp. 989-1000, 2013.

[4] P.F. Chen, C. Lee, and J.H. Zeng, "The relationship between spot and futures oil prices: Do structural breaks matter?," Energy Economics, vol. 43, pp. 206-217, 2014.

[5] G. Guo, and Z. Zheng, "The impact of international energy price shocks on China's stock market-econometric model-based empiri- cal tests," China Industrial Economics, vol. 2011, no. 6, pp. 26-35, 2011.

[6] X. Zhou, Q. Li, and B. Zhang, "World oil price risk measurementbased on the EGARCH-EVT-t Copula model," Journal of Beijing Institute of Technology, vol. 2012, no. 8, pp. 10-16, 2012.

[7] C. Xie, M. Qu, and G. Wang, "A study on most effective hedge ratio in the gold market based on the M-Copula-GJR-VaR model," Management Science, vol. 2013, no. 4, pp. 90-99, 2013.

[8] T. Bollerslev, "Generalized autoregressive conditional heteroskedasticity," Journal of Econometrics, vol. 31, pp. 307-327, 1986.

[9] A.J. Patton, "Modeling asymmetric exchange rate dependence," International Economics Review, vol. 47, pp. 527-556, 2006.

[10] X. Chen, and Y. Fan, "Estimation of Copula-based semiparametric time series models," Journal of Econometrics, vol. 130, pp. 307$335,2006$.

[11] X. Chen, W.B. Wu, and Y. Yi, "Efficient estimation of copulabased semiparametric Markov models," Annals of Statistics, vol. 37, pp. 4214-4253, 2009.

(C) Xin and Jun; Licensee Bentham Open.

This is an open access article licensed under the terms of the (https://creativecommons.org/licenses/by/4.0/legalcode), which permits unrestricted, noncommercial use, distribution and reproduction in any medium, provided the work is properly cited. 